\title{
Cold Exposure Differentially Stimulates Angiogenesis in BAT and WAT of Mice: Implication in Adrenergic Activation
}

\author{
Xiao Luo ${ }^{a, b}$ Ru Jia ${ }^{a, b, c}$ Xiao-qin Luo ${ }^{d}$ Guan Wang ${ }^{\text {e }}$ Qiang-ling Zhang ${ }^{a, b}$ \\ Hu Qiao ${ }^{a, b}$ Nan Wang ${ }^{a, b}$ Jian-qun Yana,b
}

aDepartment of Physiology and Pathophysiology, School of Basic Medical Sciences, Xi'an Jiaotong University Health Science Center, Xi'an, 'bey Laboratory of Environment and Genes Related to Diseases (Xi'an Jiaotong University), Ministry of Education of China, 'Department of Prosthodontics, Stomatological Hospital, College of Stomatology, Xi'an Jiaotong University, Xi'an, dDepartment of Medicine, School of Public Health, Xi'an Jiaotong University Health Science Center, Xi'an, China; eDana-Farber Cancer Institute of Harvard Medical School, Harvard University, Boston, USA

\section{Key Words}

Angiogenesis $\cdot$ Adipose tissues $\cdot$ Cold exposure $\cdot$ Time course $\cdot$ Adrenergic activation

\begin{abstract}
Background/Aims: To characterize the temporal profile of cold-induced angiogenesis in brown and white adipose tissues of mice in vivo and the temporal changes of angiogenic factors in primary mice brown (BA) and white adipocytes (WA) treated with $\beta_{3}$-adrenoceptor agonist $(\mathrm{CL} 316,243)$ in vitro. Methods: 8 -week old male C57BL/6J mice were individually housed in conventional cages under cold exposure $\left(4^{\circ} \mathrm{C}\right)$ for $1,2,3,4$ and 5 days. Interscapular brown adipose tissue (iBAT), inguinal subcutaneous (sWAT) and epididymal white adipose tissues (eWAT) were harvested for immunohistochemical and gene expression analysis. In vitro, primary mice BA and WA treated with or without CL316,243 were harvested for gene expression and protein secretion analysis. Results: A combination of morphological and genetic (Vegfa, Vegfr2, Hif-1 $\alpha$, Pail and Pedf) analyses demonstrated depot-specific angiogenesis in response to cold exposure. Upon CL316,243 treatment, angiogenic factors (Vegfa, Vegfr2, Hif$1 \alpha$, Pail and Pedf) and secreted protein VEGFA were transiently increased in both BA and WA. Conclusion: Our results show that iBAT is highly responsive to cold-induced angiogenesis that is mainly supported by sWAT with a lesser extent by eWAT. Moreover, the angiogenesis is a transient process with the angiogenic factors may work in an autocrine/paracrine manner.
\end{abstract}

(C) 2017 The Author(s)

Published by S. Karger AG, Basel

\section{Introduction}

Adipose tissue is the most plastic tissue in the body that undergoes constant remodeling throughout lifetime depending on the metabolic status. White and brown $\mathrm{X}$. Luo and R. Jia contributed equally to this work. 
adipose tissues (WAT and BAT) plasticity, namely the ability to expand, plays a critical role in the regulation of body energy balance. In human beings, WAT overexpansion may lead to obesity and is associated with multiple metabolic complications including type 2 diabetes and cardiovascular diseases [1,2]. Meanwhile, increased BAT expandability/plasticity has been shown to be an important factor referring to healthy metabolic profile $[3,4]$. Therefore, there is an unmet need for understanding the cellular and molecular mechanisms involved in adipose tissue plasticity.

Adipose tissue is highly vascularized and each adipocyte is encircled by plentiful capillary network, supporting adipose tissue plasticity $[5,6]$. The vasculature provides correct oxygen tension, nutrients, hormones and growth factors to adipose tissue, meanwhile, removing metabolic wastes $[7,8]$. The vessels also supply progenitor cells that can differentiate into preadipocytes and vascular endothelial cells [7]. Besides, activated endothelial cells in adipose tissue produce multifarious cytokines and growth factors regulating adipogenesis, which is the main part of adipose tissue plasticity $[9,10]$. To adapt to the changes in volume and metabolic rate of adipose tissue, adipose vasculature requires constant modulation by several angiogenic regulators. Adipocytes themselves are able to regulate angiogenesis by adipokines secretion [11]. As the largest endocrine gland in human body, adipose tissues produce several pro-angiogenic factors such as vascular endothelial growth factor A (VEGFA), as well as anti-angiogenic factors including thrombospondin-1 (TSP-1), or other angiogenic regulators like plasminogen activator inhibitor 1 (PAI-1) $[10,12,13]$. These angiogenic factors play comprehensive roles in regulating angiogenesis.

It is widely believed that overexpanded WAT is associated with obesity related morbidities. In contrast, as a thermogenic organ, the activity of BAT counteracts the obesity. BAT possesses extraordinarily complex vascular networks to support its high thermogenic activity. It also meets the needs of supplying oxygen and substrates to fuel thermogenesis and distributing heat throughout the body rapidly [14]. Although the primary role of BAT is to generate heat, brown adipocytes function similarly as white adipocytes and secrete adipokines including angiogenic factors that regulate the neovascularization, maintenance and remodeling of the vasculature [15]. The presence of metabolically active BAT in adult human has attracted extensive interests in recent years [16]. It has been demonstrated that acute cold exposure can activate BAT in human, which exhibits considerable plasticity and simultaneously activated angiogenesis $[17,18]$. In addition, recent studies showed that subcutaneous WAT (sWAT) can be converted into BAT under certain circumstances such as prolonged cold exposure or $\beta_{3}$-adrenoceptor agonist treatment [19]. The concept of this process, termed "browning" of white adipocytes, has emerged with the recognition that there is a second type of brown adipocyte within sWAT - the beige or brite adipocyte [20]. Beige adipogenesis may be accompanied by switching on an angiogenic phenotype. In several rodent models, induced pro-angiogenic factors have been detected in BAT following several hours of cold exposure [21,22]. Furthermore, typical angiogenic features have been observed in BAT and sWAT after one week or longer cold exposure [12]. However, the characteristics of dynamic cold-induced angiogenesis in BAT and WAT remain largely unknown.

Our group recently reported that iBAT expansion occurs in mice from the third day of cold treatment, whilst sWAT and eWAT mass recover at day 4 of cold exposure. It implies that the adipose tissue angiogenesis is detectable during this stage. Additionally, the expression profile dynamics of the major angiogenic factors during cold exposure remain unclear. Therefore, there is a necessity to clarify the temporal profile of adipose tissue angiogenesis during cold exposure.

In this study, we have characterized the angiogenesis in iBAT, sWAT and eWAT of male C57BL/6J mice in response to a time course of cold exposure (1-5 days). We have also examined the expression of angiogenic factors in primary mice BA and WA during CL316,243 treatment. 


\section{Cellular Physiology Cell Physiol Biochem 2017;42:974-986 \begin{tabular}{ll|l} 
DOI: 10.1159/000478680 & O 2017 The Author(s). Published by S. Karger AG, Basel \\
www.karger.com/cpb
\end{tabular} \\ Luo et al.: Angiogenesis in Brown and White Adipose Tissues}

\section{Materials and Methods}

\section{Animals, Cold exposure and Tissue Collection}

All animal studies have been approved by the Ethical Committee of Xi'an Jiaotong University. The procedures involving animals were performed as described previously. In brief, male C57BL/6J mice at 8 weeks old were divided into several experimental groups. Some mice were adapted at $18^{\circ} \mathrm{C}$ for one week before being transferred to $4^{\circ} \mathrm{C}$ for $1-5$ days (Cold mice). Other mice were maintained at room temperature $\left(23^{\circ} \mathrm{C}\right.$ ) for 5 days (RT mice).

The blood was collected by retro-orbital bleeding under isoflurane anesthesia. Mice were subsequently euthanized by cervical dislocation. The iBAT, inguinal sWAT and eWAT tissues were dissected out immediately. For RNA extraction, adipose tissues were stored at $-80^{\circ} \mathrm{C}$ until further use. For immumohistochemical analysis, tissues were transferred into $4 \%$ paraformaldehyde, followed by embedding in paraffin.

\section{qRT-PCR analysis}

Real-time PCR was performed as previously described [23]. All the primers were ordered from AUGCT (Beijing, China), of which the sequences are shown in Table 1.

\section{Immunohistochemistry}

Immunohistochemistry of CD31 and vWF was performed as previously described [24]. Primary antibodies of CD31 were used at 1:300 and VWF at 1:500 (R\&D System, USA), respectively. Photos at 400x magnification were all divided into 20 fields equally and numbered uniformly. According to the random number table, six fields per mouse were chosen for data collection and analysis using ImageJ software (NIH, Bethesda, MD, USA), with six mice in each group.

Primary mice preadiopocytes culture

Primary mice brown and white preadipocytes were isolated from iBAT and inguinal sWAT of 3-weekold C57BL/6J male mice. Brown preadipocytes were cultured in DMEM (Gibco BRL, MD, USA) containing $20 \%$ fetal bovine serum (Gibco), whilst white preadipocytes were maintained in DMEM/F12 1:1 (Gibco) containing $10 \%$ fetal bovine serum at $37{ }^{\circ} \mathrm{C}$ in a humidified $5 \% \mathrm{CO}_{2}$ incubator. Cells were used for experiments at passage two after 1-2 weeks of culture.

To induce preadipocyte differentiation, cells were grown to postconfluence then cultured in the presence of a chemically defined medium [25]. After 9 days of culture in the differentiation medium, the cells had a fully differentiated phenotype with a large number of lipid accumulation.

\section{Oil red $O$ staining}

Lipid accumulation was assessed by Oil Red 0 staining. The process was performed as previously described [23]. In brief, after 9 days of differentiation, cells (in 24-well plates) were washed twice in PBS and fixed in $4 \%$ paraformaldehyde for $1 \mathrm{~h}$ in room temperature. After rinsing in 60\% isopropanol, cells were incubated in Oil red $\mathrm{O}$ working solution for $15 \mathrm{~min}$. Then cells were rinsed three times in $\mathrm{ddH}_{2} \mathrm{O}$ and photographed by phase-contrast microscopy (Nikon Eclipse TS100).

\section{Treatment of brown and white adipocytes with CL316,243}

To stimulate the thermogenic program, differentiated cells were incubated in the serum-free medium for $12 \mathrm{~h}$ prior to treatment with or without CL316,243 (0.5-2 $\mu \mathrm{M}$, Tocris Bioscience, Bristol, UK) for indicated lengths of time.

Table 1. Primers used for quantitative real-time PCR analysis

\begin{tabular}{lll}
\hline Gene & Forward primer & Reverse primer \\
\hline Vegfa & TATTCAGCGGACTCACCAGC & AACCAACCTCTCAAACCGT \\
Vegfr 2 & GATGCAGGAAACTACACGGTCA & GAGATCAAGGCTTTCTCACCGA \\
Hif-1a & GCACTAGACAAAGTTCACCTGAGA & CGCTATCCACATCAAAGCAA \\
Pai 1 & ACAGCCTTTGTCATCTCAGCC & CCGAACCACAAAGAGAAAGGA \\
Pedf & TCGAAAGCAGCCCTGTGTT & AATCACCCGACTTCAGCAAGA \\
Cyclophlin & CATACAGGTCCTGGCATCTTGTC & AGACCACATGCTTGCCATCCAG \\
\hline
\end{tabular}




\section{Cellular Physiology Cell Physiol Biochem 2017;42:974-986 \begin{tabular}{ll|l} 
and Biochemistry Published onlıne: June 27, 2017 & $\begin{array}{l}\text { C } 2017 \text { The Author(s). Published by S. Karger AG, Basel } \\
\text { www.karger.com/cpb }\end{array}$
\end{tabular} \\ Luo et al.: Angiogenesis in Brown and White Adipose Tissues}

VEGFA and PAI1 secretion

Mice plasma and conditioned medium from brown and white adipocytes were collected at the indicated time points. VEGFA and PAI1 concentrations were determined by ELISA (Thermo Fisher Scientific, USA).

\section{Statistical analysis}

Data were expressed as means \pm S.E.M. of independent experiments and statistical analyses were performed in GraphPad Prism 6.0 using the following tests: Student's $t$ test for differences between two experimental groups, One-way ANOVA for differences across experimental groups in conjunction with Tukey post hoc test to compare differences between treatment groups. $P<0.05$ was considered as statistically significant.

\section{Results}

Cold exposure induces angiogenesis in iBAT and sWAT, but not in eWAT

To determine the potential effects of cold exposure on angiogenesis in BAT and WAT of mice, experiments were performed to compare mice maintained at room temperature (RT) or cold-exposed (Cold) environment for up to five days. Histological analysis revealed higher number of CD31-positive cells in iBAT and sWAT of Cold mice at days 4-5 and day 5 respectively than those at RT, indicating a larger number of microvessels in iBAT and sWAT of Cold mice (Fig. 1A). Intriguingly, although the eWAT adipocyte size of Cold mice appeared smaller than those at RT, no obvious difference in the number of CD31-positive cells between Cold and RT mice had been observed (Fig. 1A). Quantitation of microvessel density confirmed these observations, manifesting that the proportion of CD31-positive area was increased in iBAT and sWAT from day 4 (Fig. 1B-C). Despite failing to reach statistical significance, the proportion of CD31-positive area in eWAT showed the tendency of increase at day 3 of cold exposure as well (Fig. 1D). The expression level of von Willebrand Factor (vWF), another angiogenesis marker, was higher than that of CD31 in adipose tissues. However, the expression pattern of vWF was as similar as that of CD31. Histological analysis showed significantly more vWF-positive cells in iBAT and sWAT of Cold mice comparing with RT mice, at day 4 and maintained thereafter (Fig. 1E-G). However, no significant difference in eWAT has been observed (Fig. 1E). Quantitation of the proportion of vWF-positive area in eWAT again confirmed these observations (Fig. 1H). These results indicate that the acute cold exposure significantly increases microvessel densities in iBAT and sWAT, but lesser impact is exerted to eWAT.

Cold exposure differentially changes the expression of angiogenic factors

As cold exposure differentially changed the microvessel density in adipose tissues, we further investigated the changes of angiogenic factors in iBAT, sWAT and eWAT. Real-time PCR results showed that the changes in the expression of the core pro-angiogenic gene, vascular endothelial growth factor A (Vegfa), differed in each of the depots (Fig. 2A). Perhaps surprisingly, in iBAT, Vegfa expression decreased by around $50 \%$ from day 2 to day 5 . In sWAT, it increased gradually from day 1 to day 3 , but only reached statistical significance on day 3 , and decreased thereafter. In eWAT, Vegfa expression increased around 10-fold from day 2 to day 5 . We also found that expression of vascular endothelial growth factor receptor 2 (Vegfr2), a key receptor of VEGF system in adipose tissues, increased within 1 day during cold stimulation, then decreased to basal level on subsequent days in all three depots (Fig. 2B). Interestingly, hypoxia-inducible transcription factor $1 \alpha($ Hif- $1 \alpha)$, a master mediator of hypoxia signal, was upregulated in iBAT and eWAT at day $1 \& 4$ and day 3 respectively with no significant changes observed in sWAT. Notably, the Hif-1 $\alpha$ levels in eWAT were 3-5fold higher than those in iBAT and sWAT (Fig. 2C). These data demonstrate that the relative expression of the pro-angiogeneic factors, the magnitude of the changes and the temporal profiles differ in all depots. 

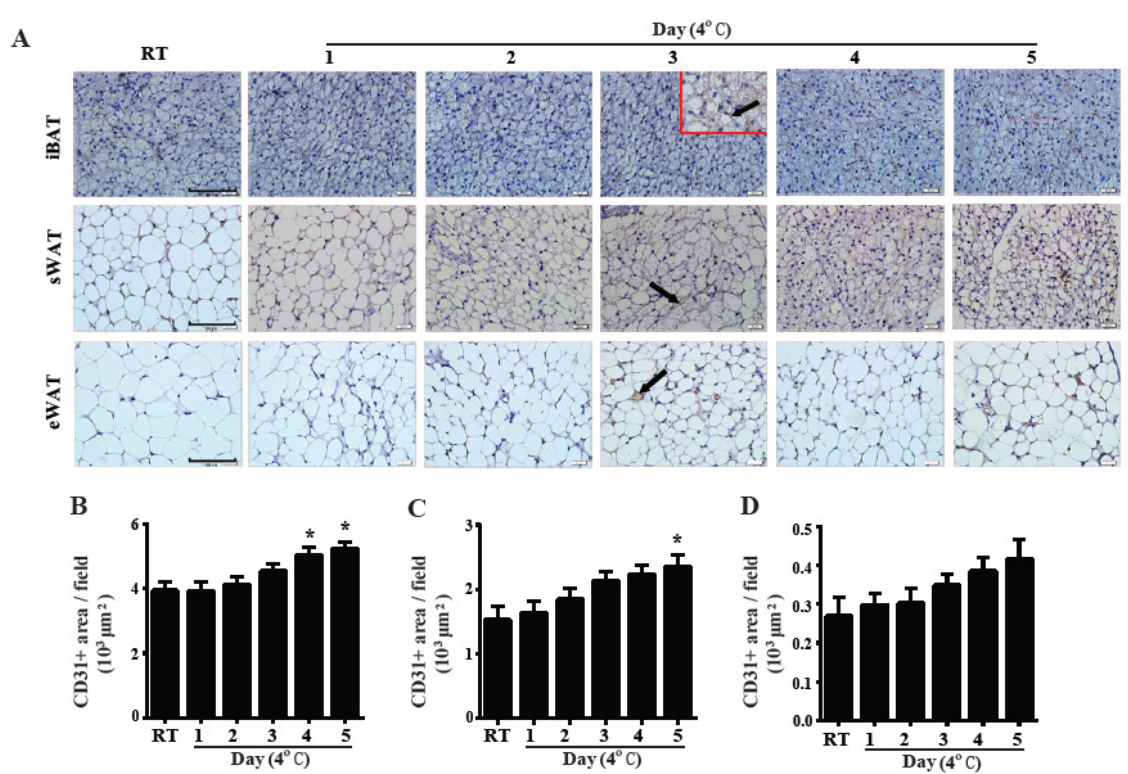

$\mathbf{E}$
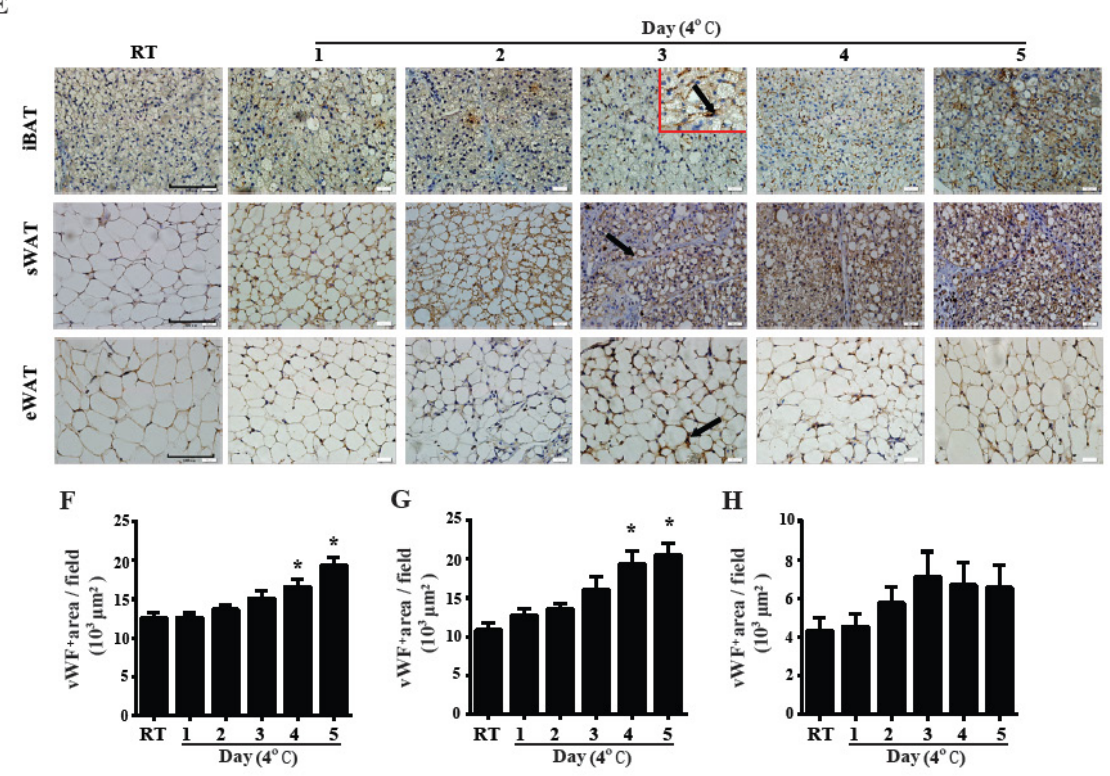

Fig. 1. Cold exposure induced angiogenesis in iBAT and sWAT, but not in eWAT. (A)Representative photomicrographs (original magnification $400 \times$, Scale bar: $100 \mu \mathrm{m}$ ) of iBAT, sWAT and eWAT sections stained with CD31 from RT and Cold mice. Quantification of CD31-positive area in iBAT (B), sWAT (C) and eWAT (D). (E) Representative photomicrographs (original magnification 400×, Scale bar: $100 \mu \mathrm{m}$ ) of iBAT, sWAT and eWAT sections stained with vWF from RT and Cold mice. Quantification of vWF-positive area in iBAT (F), sWAT (G) and eWAT (H). Data were collected and analyzed from CD31- and vWF-stained sections of six individual mice in each group, six random fields per mouse, 20-40 cells per field at 400×magnification, using Image J software (NIH, Bethesda, MD, USA). Solid arrows point to positive staining (brown). Values are mean \pm S.E.M. $\left({ }^{*} P<0.05\right)$.

Having observed the temporal depot-specific induction profiles of pro-angiogenic factors following cold exposure, we extended our investigation to examine the antiangiogenic factors. Interestingly, the expression levels of plasminogen activator inhibitor 1 (Pai1), which is involved in vessel destabilisation, differed markedly across the depots (Fig. 2D). In iBAT, the Pai1 levels increased around 2-fold on day 2 before dropping down to below 
Fig. 2. Cold exposure differentially changed the expression of angiogenic factors. Quantitative PCR analysis of Vegfa (A), Vegfr2 (B), Hif-1 $\alpha$ (C), Pai1 (D) and Pedf (E) gene expression in iBAT, sWAT and eWAT of RT mice and Cold mice for up to $5 \mathrm{~d}$. Data are expressed as fold compared mice respectively. Values are mean \pm S.E.M. and expression of genes is corrected for the housekeeping gene Cyclophilin. $(\mathrm{n}=6$ for each group, ${ }^{*} P<0.05$, $* * P<0.01)$. with iBAT of RT

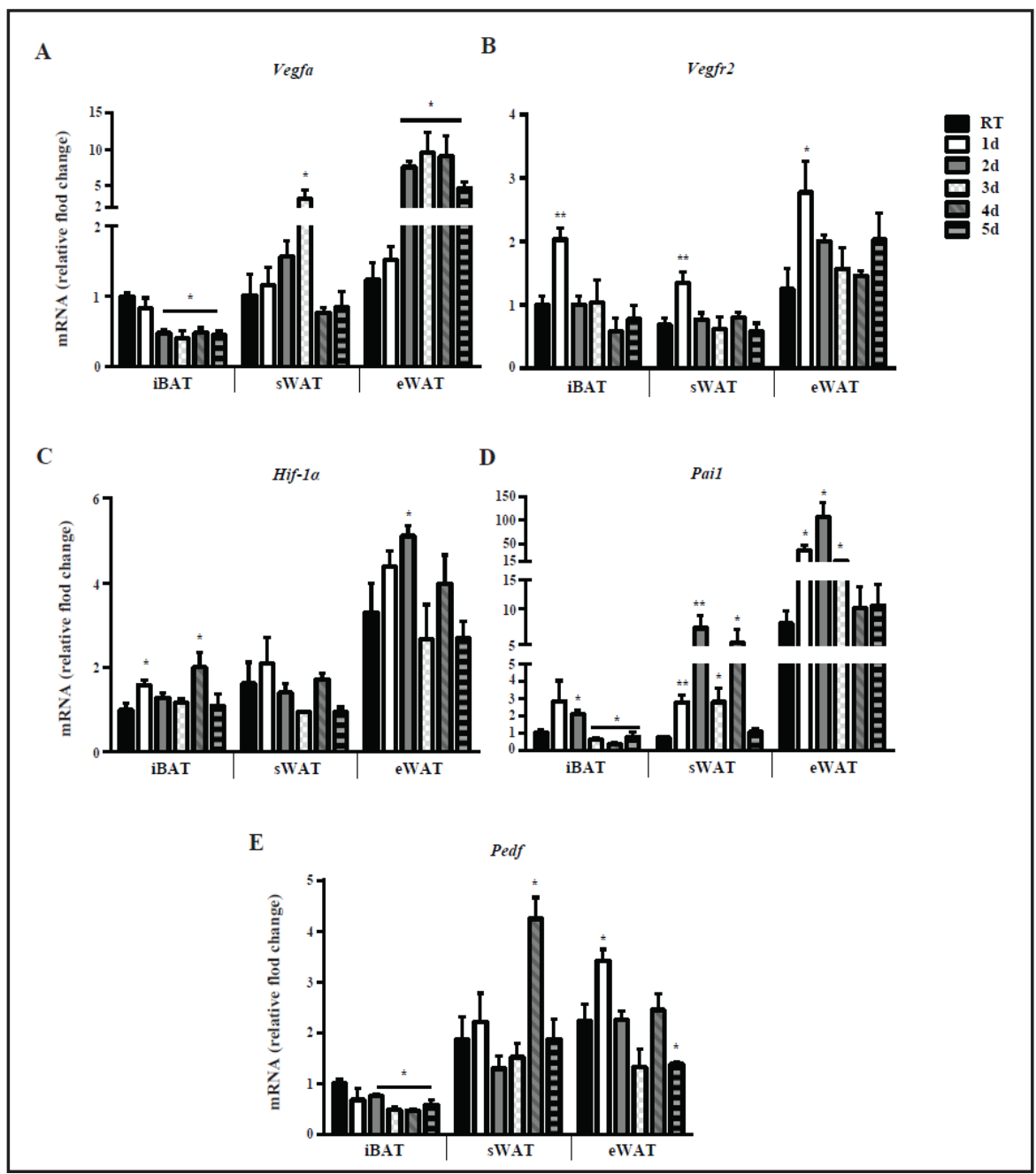

Fig. 3. CL316,243 stimulation of $U c p-1$ and $P g c-1 \alpha$ gene expression in BA and WA. (A) Micrographs showing Oil Red 0 staining of mature adipocytes on day 9 of differentiation. Quantitative PCR analysis of Ucp-1 (B) and Pgc-1 $\alpha$ (C) gene expression in mature BA and WA after treatment with indicated dose of CL for $6 \mathrm{~h}$. Data are expressed as fold compared with zero groups respectively. Values are mean \pm S.E.M. from three independent experiments with duplicate wells for each dose of treatment.

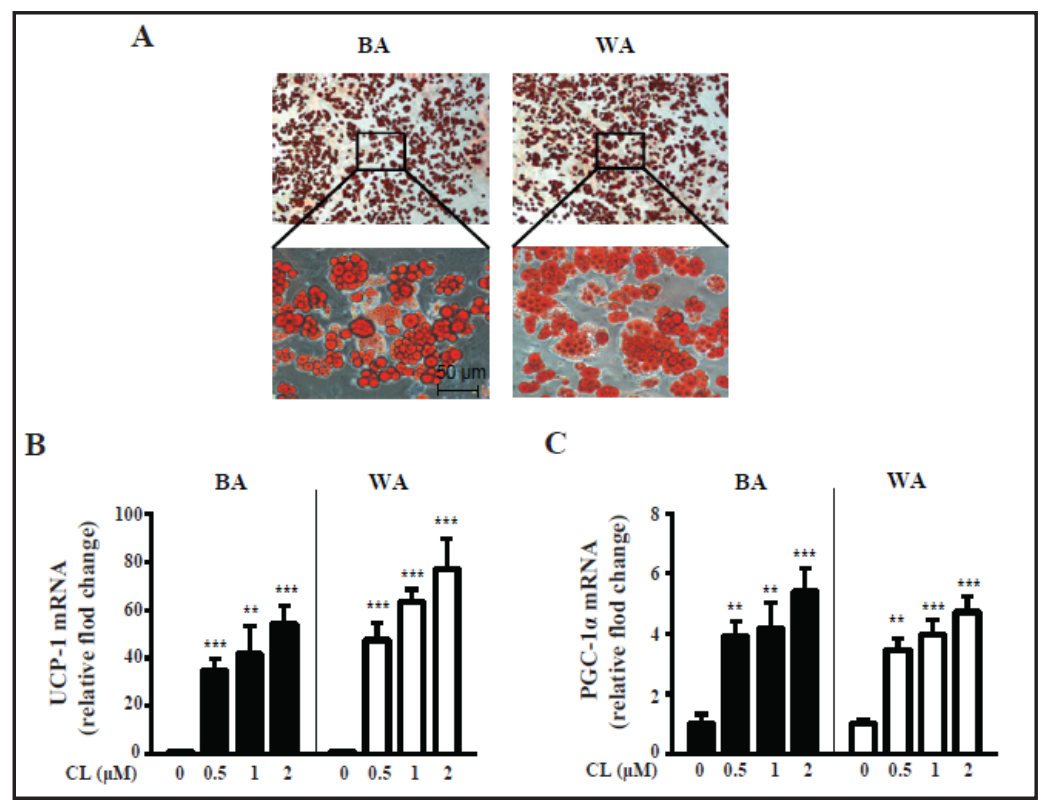

The expression of genes is corrected for the housekeeping gene Cyclophilin. $\left({ }^{* *} P<0.01,{ }^{* * *} P<0.001\right)$ CL: CL316,243; BA: brown adipocytes; WA: white adipocytes. 


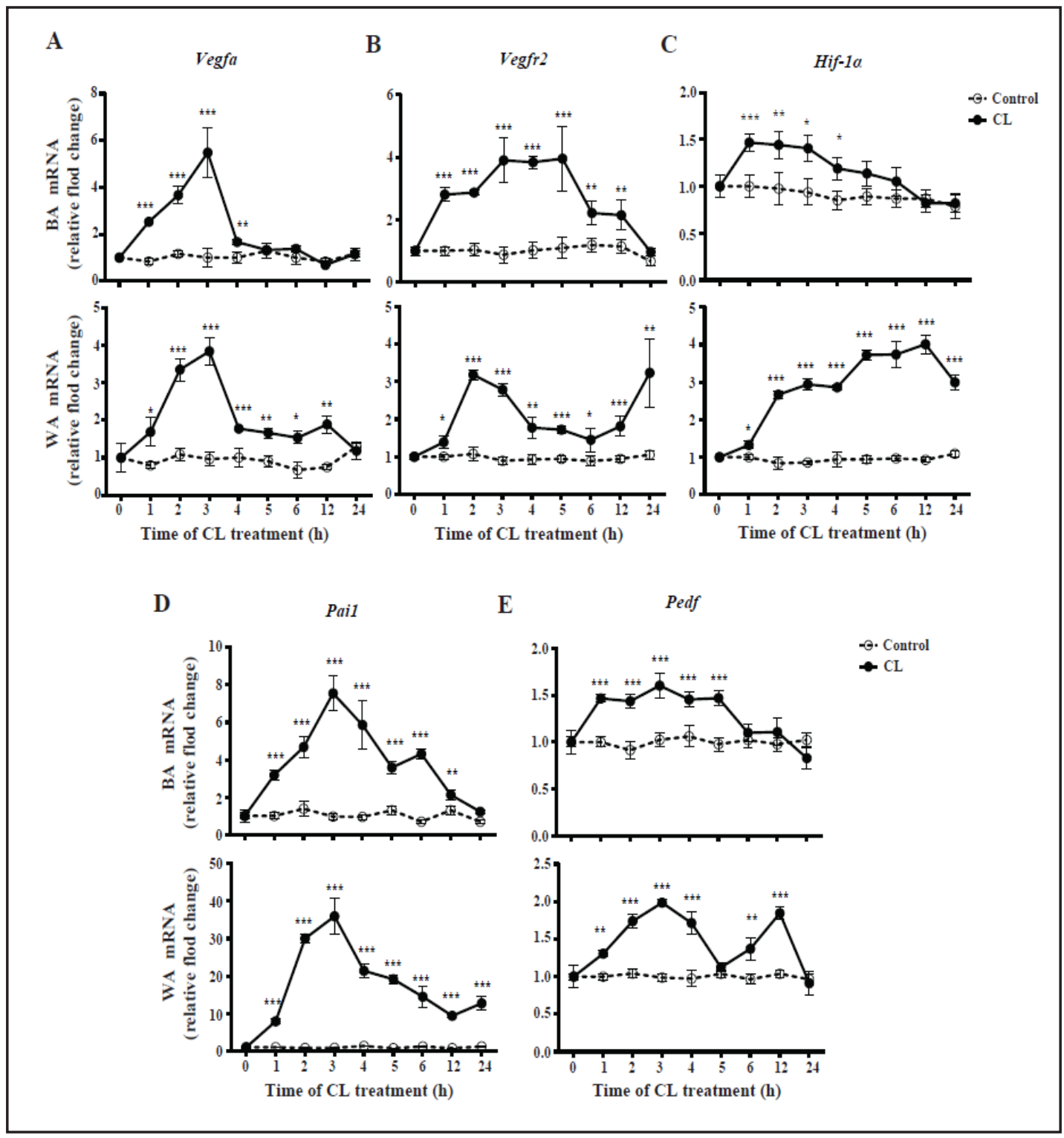

Fig. 4. CL316,243 treatment induced the expression of angiogenic factors in BA and WA . Quantitative PCR analysis of Vegfa (A), Vegfr2 (B), Hif-1 $\alpha$ (C), Pai1 (D) and Pedf (E) gene expression in BA and WA after treatment with $2 \mu \mathrm{M}$ CL for 0-24 h. Data are expressed as fold compared with control group at each time point. Values are mean \pm S.E.M. from three independent experiments with duplicate wells for each time of treatment. The expression of genes is corrected for the housekeeping gene Cyclophilin. $\left({ }^{*} P<0.05,{ }^{* *} P<0.01\right.$, *** $P<0.001)$.

the baseline on days 3-5. In contrast, the Pai1 levels in sWAT increased to 3-7-fold from day 1 before returning to the basal level on day 5. Notably, despite its high baseline, the Pai1 levels in eWAT increased dramatically on days 1-3 to reach about 7-fold higher than those in iBAT and sWAT (Fig. 2D). The expression levels of another anti-angiogenic marker, pigment epithelium derived factor (Pedf), decreased by $50 \%$ in iBAT from day 2 onwards (Fig. 2E). However, in sWAT and eWAT, cold exposure only induced transient increases of Pedf at day 4 and day 1 respectively (Fig. 2E). Collectively, profiling cold-induced angiogenic markers expression demonstrates that the angiogenesis of adipose tissues in response to cold exposure appears to be a depot-specific manner. 
A

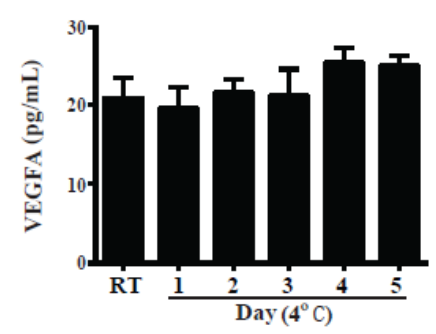

C

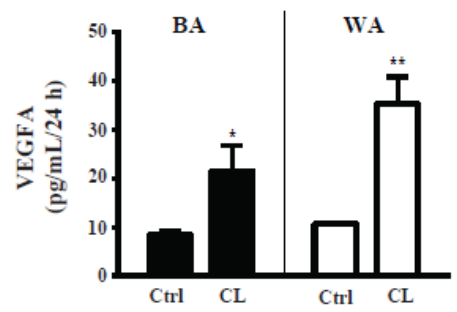

B

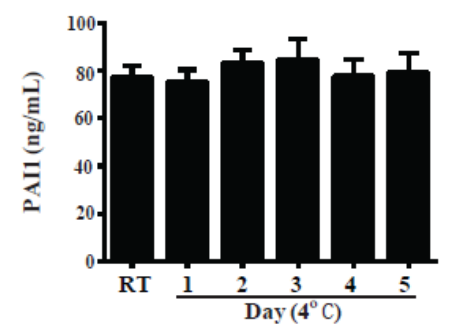

D

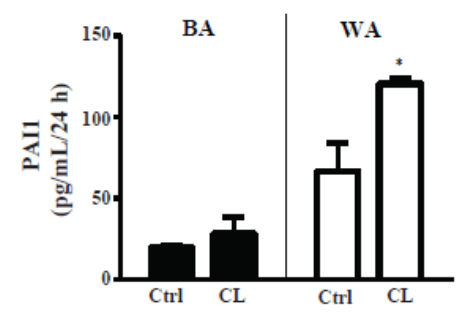

Fig. 5. CL316,243 treatment induced the VEGFA and PAI1 secretion. VEGFA (A) and PAI1 (B) secretion in plasma were measured by ELISA. Values are mean \pm S.E.M. ( $n=6$ for each group). VEGFA (C) and PAI1 (D) secretion in cultured medium were measured by ELISA. Results show mean \pm S.E.M. from three to four independent experiments. $\left({ }^{*} P<0.05,{ }^{* *} P<0.01\right.$ comparing control with $2 \mu \mathrm{M}$ CL treatment).

CL316,243 treatment induces the expression of angiogenic factors in primary brown and white adipocytes

It has been well established that cold-induced adipose tissue remodeling and browning was involved in activation of $\beta_{3}$ AR-cAMP pathway in vivo. Therefore, after figuring out the temporal details of depot-specific angiogenesis induced by cold exposure, we investigated whether the activation of $\beta_{3}$-adrenoceptor directly effected on the changes of angiogenic factors expression in adipocytes. Primary cultures of mice BA and WA isolated from iBAT and sWAT were utilized to verify our hypothesis. As shown in Fig. 3A, most preadipocytes were filled with lots of lipid after 9 days of differentiation. Indeed, we found the treatment of the BA and WA with different doses of $\beta_{3}$-adrenoceptor agonist, CL316,243 (CL), induced a rapid and sustained up-regulation of the core thermogenic gene, uncoupling protein 1 (Ucp1). Although insignificant, Ucp1 expression showed a tendency of dose-dependent upregulation by CL treatment (Fig. 3B). We also found $\alpha$ subunit of peroxisome proliferatorsactivated receptor- $\gamma$ coactivator $1(P g c-1 \alpha)$, a key inducer of brown adipocyte activation, showed a similar expression pattern to Ucp1 in both BA and WA (Fig. 3C). These results imply that the $\beta_{3} A R$-cAMP pathway may be involved in the expression of the browning markers in $\mathrm{BA}$ and WA.

We further investigated whether the $\beta_{3}$ AR-cAMP pathway was indeed associated with the changes of angiogenic factors expression. As expected, CL treatment induced a transient increase in expression of Vegfa in BA at hours 1-4. In WA, the expression of Vegfa was rapidly induced (peaking at around 5-fold at hour 3) before decreasing to basal level at hour 24 (Fig. 4A). Rapid induction of Vegfr2 expression by CL was also observed in BA and WA, peaking at hour 3 and hour 2 respectively (Fig. 4B). Interestingly, over the 24-hour timecourse, CL treatment increased Hif-1 $\alpha$ expression significantly at all time points in WA while only induced significant increases from hour 1-4 in BA (Fig. 4C). To our surprise, the anti-angiogenic marker, Pai1, showed an expression pattern similar to Vegfr2 in both BA and WA (Fig. 4D). The expression levels of pedf, another anti-angiogenic marker, also transiently increased at hours 1-5 before returning to the baseline in BA. In contrast, in WA, pedf levels were rapidly induced except for hour 5 and hour 24, at which pedf levels dropped back to baseline (Fig. 4E). 
To extend our observations at the gene level we characterized the effects of cold exposure and CL on changes of the secretory proteins VEGFA and PAI1. Unexpectedly, no obvious differences have been observed in both VEGFA and PAI1 proteins between RT and Cold mice plasma (Fig. 5 A-B). Nevertheless, compared to the control cells, CL treated cells secreted more VEGFA protein in BA and WA, with around 2-fold and 4-fold of increases respectively (Fig. $5 \mathrm{C}$ ). Although no change has been observed in BA, the secretion level of PAI1 showed around 2-fold of increase in WA (Fig. 5 D). The differences of CL-induced angiogenic factors expression further support the notion that cold-induced adipose tissue angiogenesis is depot-specific.

\section{Discussion}

It is well established that the plasticity of adipose tissue requires its vascularization via the processes of angiogenesis. Recently, inappropriate angiogenesis has been shown to underlie certain adipose tissue dysfunction in obesity, which in turn increases the metabolic complications risk $[5,26]$. Therefore, understanding and controlling the angiogenesis in adipose tissues may offer a great opportunity to the treatment of obesity and metabolic disorders. Several murine studies have shown that acute cold acclimation or $\beta_{3}$-adrenergic agonists induces pro-angiogenic factors in BAT, whilst prolonged exposure of mice to cold results in activation of angiogenesis in iBAT and sWAT [12, 21, 22, 27]. We have recently reported that the remodeling and browning in BAT and WAT during cold exposure exhibit depot-specific differences [28]. However, it remains elusive to what extent BAT and WAT angiogenesis occurs following cold exposure, and whether it shows any depotspecific differences. In this study, we have addressed this by characterizing the profiles of angiogenesis during 1-5 days cold exposure in mouse BAT and WAT and the temporal changes of angiogenic factors in mouse BA and WA following CL treatment. We have shown that BAT and WAT responded differently to cold-induced angiogenesis in vivo. In addition, by utilizing primary brown and white adipocytes, we have observed that the CL treatment rapidly increased the expression of angiogenic factors.

Because of the extremely high demand for blood flow during active thermogenesis, BAT possesses extensive microvessel network. We found that iBAT had a higher degree of vascularization than sWAT and eWAT in RT mice, principally in agreement with previous observations $[12,29]$. Recent studies have also shown that prolonged periods of cold exposure increases BAT capillary density [12]. Consistent with this, we observed a significant increase in iBAT microvessel density of Cold mice after 4 days. Interestingly, this timepoint happens to coincide with our recent report, which implies days 4-5 after cold exposure is the 'cold adapted' phase of adipose tissues remodeling [28] . It is tempting to speculate that the induced angiogenesis in iBAT may contribute to the major benefits of the 'cold adapted' phase. Even though further studies are required to verify this hypothesis, it is believed that a new steady-state level of vascularization needs to be established for new energy demands on adipose tissues during 'cold adapted' phase. Although thermogenesis and sympathetic activity are constant during cold exposure, previous studies have indicated the phase of induced angiogenesis is transient [12,21,27]. Our findings of the transient increases of proangiogenesis factors Vegfr2 and Hif-1 $\alpha$ both in vivo and in vitro support this notion. In addition, we observed a rapid increase in the mRNA level of pro-angiogenesis factor Vegfa upon 1-4 $\mathrm{h}$ CL stimulation in BA, which is consistent with other studies that demonstrated a transient induction in Vegfa expression following norepinephrine treatment [21, 27, 30]. Intriguingly, the Vegfa level exhibited a significant decrease after 2 days of cold exposure. This phenotype may be associated with a "switching-off" mechanism of cold-induced angiogenesis to prevent over-neovascularization in BAT, which is an interesting question worth further investigations. Adipose tissue angiogenesis is at least partially regulated by the balanced release of proand anti-angiogenic proteins secreted from adipocytes. PAI1 and PEDF have been shown as the inhibitors of angiogenesis process [31, 32]. However, a recent report described an 
inexplicable finding that overexpression of PEDF did not alter angiogenesis in mice adipose tissue [33]. Moreover, PAI1 has been reported to both positively and negatively regulate angiogenesis depending on the dosages [34]. These reports demonstrated the complex roles of PAI1 and PEDF in adipose tissue angiogenesis. Our studies support this view when the expression patterns of Pail and Pedf in BAT are compared with those in cultured BA (for example, Pedf expression decreased in BAT but transiently increased in BA).

It has been reported that the adipose tissue remodeling, relevant to beige adipogenesis, becomes more prominent within sWAT in mice after cold exposure or $\beta_{3}$-adrenoceptor agonist treatment [35]. Within this phase, the angiogenesis is simultaneously activated [12]. Consistent with this, our study showed 5 days of cold exposure induced angiogenesis of sWAT, indicated by an increase in the CD31- and vWF-positive cell staining with subsequent increases in expression of Vegfa and Vegfr2, the markers of angiogenesis. Additionally, our in vitro study showed the pro-angiogenesis factors Vegfa, Vegfr2, Hif-1 $\alpha$ and secreted protein VEGFA were up-regulated rapidly following the CL treatment. Interestingly, we also observed significant increases of Pai1 and Pedf in sWAT from days 1-4 and day 4 of cold exposure respectively, as well as in cultured WA following CL treatment. Given that PAI1 plays opposite roles in angiogenesis depending on the dosages [34], we infer that the increased Pai1 mRNA may result in the production of low dose of PAI1 protein to induce angiogenesis in sWAT. Further studies are needed to determine the concentration of PAI1 contributing to coldinduced sWAT angiogenesis. Moreover, Lakeland et al. has reported that instead of altering angiogenesis, the predominant role of PEDF is to modulate lipolysis positively [33]. It is possible that the cold exposure and CL treatment in sWAT utilize this mechanism to provide a fuel substrate to meet the increased energy demands.

We recently reported that the remodeling of eWAT is very modest upon cold exposure [28]. In this study, we also observed that eWAT was relatively non-responsive to cold-induced angiogenesis, indicated by the unaltered numbers of the CD31- and vWF-positive cells. Nevertheless, the major angiogenic factors were significantly increased in eWAT during cold exposure. Moreover, our finding of higher levels of Vegfa and Pai1 in eWAT compared to iBAT and sWAT were in line with previous studies which reported higher expressions of Vegfa and Pai1 in omentum [36, 37]. Intriguingly, despite the high expression of angiogenic factors, the angiogenesis in eWAT was relatively chronic and mild responding to cold exposure. Collectively, these observations demonstrated that the mechanisms of angiogenesis among different depots may vary significantly.

We noticed that some angiogenic genes such as Vegfa and Pedf, exhibited different expression pattern between experiments employing in vivo cold exposure and in vitro CL treatment. This may be due to the different observation time of the experiments in vitro and in vivo. In addition, our in vivo experiments suggest that angiogenic genes are not merely regulated by $\beta_{3}$ AR-cAMP pathway. Further studies are in need to uncover the molecular mechanism for the regulation of angiogenic genes in adipose tissue.

It is well known that most adipokines play the regulatory roles in the manner of autocrine, paracrine or endocrine [38]. Nicosia et al. reported that VEGF regulates rat aorta angiogenesis in an autocrine/paracrine manner [39]. A recent study also provided evidence for a VEGF-mediated autocrine/paracrine regulation of stem cell regeneration [40]. Dohgu et al. showed PAI1 up-regulates the blood-brain barrier function in the ways of autocrine and paracrine [41]. Our findings of no changes in VEGFA and PAI1 levels in plasma but significant increases in cultured medium reinforce the notion that the main regulatory manner of these angiogenic factors is autocrine or paracrine. Therefore, more investigations are in need to determine the regulatory mechanisms for the secreted levels of angiogenic factors in adipose tissues.

In summary, the presented data demonstrate that BAT and WAT display dynamic alterations in terms of angiogenic characteristics and the expression of angiogenic factors during cold exposure. In order to significantly contribute to the thermogenic process throughout cold acclimation, iBAT angiogenesis rapidly increases in the early stage after cold exposure, and is partially supported by the angiogenesis of sWAT, and to a lesser extent

\section{KARGER}




\section{Cellular Physiology Cell Physiol Biochem 2017;42:974-986

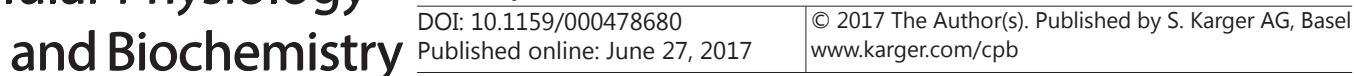 \\ Luo et al.: Angiogenesis in Brown and White Adipose Tissues}

of eWAT. In addition, we show the expression patterns of angiogenic factors in adipose tissues are transient and their regulatory manners are mainly autocrine and paracrine. This notion is also supported by the dynamic changes in angiogenic factors in primary culture experiments. To determine how the angiogenic factors transiently express upon the cold stimulation remains a challenge for future studies.

\section{Acknowledgments}

This work was supported by fundings from the National Natural Science Foundation of China (31200886), the China Postdoctoral Science Foundation (2015M580856), the Natural Science Foundation of Shaanxi Province (2016JQ8021) and the Science Foundation for Postdoctoral Scientists of Shaanxi Province.

\section{Disclosure Statement}

No potential conflicts of interest relevant to this article were disclosed.

\section{References}

1 Dubois SG, Heilbronn LK, Smith SR, Albu JB, Kelley DE, Ravussin E, Look AARG: Decreased expression of adipogenic genes in obese subjects with type 2 diabetes. Obesity 2006;14:1543-1552.

-2 Spalding KL, Arner E, Westermark PO, Bernard S, Buchholz BA, Bergmann O, Blomqvist L, Hoffstedt J, Naslund E, Britton T, Concha H, Hassan M, Ryden M, Frisen J, Arner P: Dynamics of fat cell turnover in humans. Nature 2008;453:783-787.

- 3 Ouellet V, Routhier-Labadie A, Bellemare W, Lakhal-Chaieb L, Turcotte E, Carpentier AC, Richard D: Outdoor temperature, age, sex, body mass index, and diabetic status determine the prevalence, mass, and glucose-uptake activity of 18f-fdg-detected bat in humans. J Clin Endocrinol Metab 2011;96:192-199.

4 Lee P, Greenfield JR: Non-pharmacological and pharmacological strategies of brown adipose tissue recruitment in humans. Mol Cell Endocrinol 2015;418 Pt 2:184-190.

5 Corvera S, Gealekman 0: Adipose tissue angiogenesis: Impact on obesity and type-2 diabetes. Biochim Biophys Acta 2014;1842:463-472.

6 Lemoine AY, Ledoux S, Larger E: Adipose tissue angiogenesis in obesity. Thromb Haemost 2013;110:661668.

7 Tang W, Zeve D, Suh JM, Bosnakovski D, Kyba M, Hammer RE, Tallquist MD, Graff JM: White fat progenitor cells reside in the adipose vasculature. Science 2008;322:583-586.

8 Hausman GJ, Richardson RL: Adipose tissue angiogenesis. J Anim Sci 2004;82:925-934.

-9 Palacios-Ortega S, Varela-Guruceaga M, Algarabel M, Ignacio Milagro F, Alfredo Martinez J, de Miguel C: Effect of tnf-alpha on caveolin-1 expression and insulin signaling during adipocyte differentiation and in mature adipocytes. Cell Physiol Biochem 2015;36:1499-1516.

10 Cao R, Brakenhielm E, Wahlestedt C, Thyberg J, Cao Y: Leptin induces vascular permeability and synergistically stimulates angiogenesis with fgf-2 and vegf. Proc Natl Acad Sci U S A 2001;98:6390-6395.

11 Cao Y: Angiogenesis modulates adipogenesis and obesity. J Clin Invest 2007;117:2362-2368.

12 Xue Y, Petrovic N, Cao R, Larsson O, Lim S, Chen S, Feldmann HM, Liang Z, Zhu Z, Nedergaard J, Cannon B, Cao Y: Hypoxia-independent angiogenesis in adipose tissues during cold acclimation. Cell Metab 2009;9:99-109.

13 Voros G, Maquoi E, Demeulemeester D, Clerx N, Collen D, Lijnen HR: Modulation of angiogenesis during adipose tissue development in murine models of obesity. Endocrinology 2005;146:4545-4554.

14 Cannon B, Nedergaard J: Brown adipose tissue: Function and physiological significance. Physiol Rev 2004;84:277-359. 


\section{Cellular Physiology Cell Physiol Biochem 2017;42:974-986

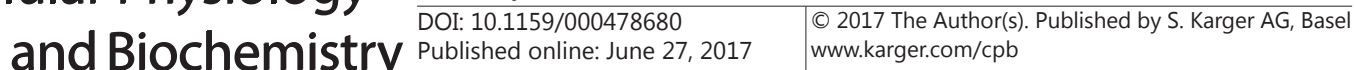

Luo et al.: Angiogenesis in Brown and White Adipose Tissues

15 Villarroya J, Cereijo R, Villarroya F: An endocrine role for brown adipose tissue? Am J Physiol Endocrinol Metab 2013;305:E567-572.

-16 Nedergaard J, Bengtsson T, Cannon B: Unexpected evidence for active brown adipose tissue in adult humans. Am J Physiol Endocrinol Metab 2007;293:E444-452.

17 Orava J, Nuutila P, Lidell ME, Oikonen V, Noponen T, Viljanen T, Scheinin M, Taittonen M, Niemi T, Enerback S, Virtanen KA: Different metabolic responses of human brown adipose tissue to activation by cold and insulin. Cell Metab 2011;14:272-279.

18 Ouellet V, Labbe SM, Blondin DP, Phoenix S, Guerin B, Haman F, Turcotte EE, Richard D, Carpentier AC: Brown adipose tissue oxidative metabolism contributes to energy expenditure during acute cold exposure in humans. J Clin Invest 2012;122:545-552.

19 Cannon B, Jacobsson A, Rehnmark S, Nedergaard J: Signal transduction in brown adipose tissue recruitment: Noradrenaline and beyond. Int J Obes Relat Metab Disord 1996;20 Suppl 3:S36-42.

20 Petrovic N, Walden TB, Shabalina IG, Timmons JA, Cannon B, Nedergaard J: Chronic peroxisome proliferator-activated receptor gamma (ppargamma) activation of epididymally derived white adipocyte cultures reveals a population of thermogenically competent, ucp1-containing adipocytes molecularly distinct from classic brown adipocytes. J Biol Chem 2010;285:7153-7164.

21 Asano A, Morimatsu M, Nikami H, Yoshida T, Saito M: Adrenergic activation of vascular endothelial growth factor mrna expression in rat brown adipose tissue: Implication in cold-induced angiogenesis. Biochem J 1997;328:179-183.

22 Fredriksson JM, Nikami H, Nedergaard J: Cold-induced expression of the vegf gene in brown adipose tissue is independent of thermogenic oxygen consumption. FEBS Lett 2005;579:5680-5684.

23 Luo X, Hutley LJ, Webster JA, Kim YH, Liu DF, Newell FS, Widberg CH, Bachmann A, Turner N, SchmitzPeiffer C, Prins JB, Yang GS, Whitehead JP: Identification of bmp and activin membrane-bound inhibitor (bambi) as a potent negative regulator of adipogenesis and modulator of autocrine/paracrine adipogenic factors. Diabetes 2012;61:124-136.

24 Luo X, Jia R, Yao Q, Xu Y, Luo Z, Luo X, Wang N: Docosahexaenoic acid attenuates adipose tissue angiogenesis and insulin resistance in high fat diet-fed middle-aged mice via a sirt1-dependent mechanism. Mol Nutr Food Res 2016;60:871-885.

25 Luo X, Jia R, Zhang Q, Sun B, Yan J: Cold-induced browning dynamically alters the expression profiles of inflammatory adipokines with tissue specificity in mice. Int J Mol Sci 2016;17

-26 Garcia-Martin R, Alexaki VI, Qin N, Rubin de Celis MF, Economopoulou M, Ziogas A, Gercken B, Kotlabova K, Phieler J, Ehrhart-Bornstein M, Bornstein SR, Eisenhofer G, Breier G, Bluher M, Hampe J, El-Armouche A, Chatzigeorgiou A, Chung KJ, Chavakis T: Adipocyte-specific hypoxia-inducible factor 2alpha deficiency exacerbates obesity-induced brown adipose tissue dysfunction and metabolic dysregulation. Mol Cell Biol 2015;36:376-393.

27 Asano A, Kimura K, Saito M: Cold-induced mrna expression of angiogenic factors in rat brown adipose tissue. J Vet Med Sci 1999;61:403-409.

-28 Jia R, Luo XQ Wang G, Lin CX, Qiao H, Wang N, Yao T, Barclay JL, Whitehead JP, Luo X, Yan JQ: Characterization of cold-induced remodelling reveals depot-specific differences across and within brown and white adipose tissues in mice. Acta Physiol (Oxf) 2016;217:311-324.

29 Shimizu I, Aprahamian T, Kikuchi R, Shimizu A, Papanicolaou KN, MacLauchlan S, Maruyama S, Walsh K: Vascular rarefaction mediates whitening of brown fat in obesity. J Clin Invest 2014;124:2099-2112.

-30 Fredriksson JM, Lindquist JM, Bronnikov GE, Nedergaard J: Norepinephrine induces vascular endothelial growth factor gene expression in brown adipocytes through a beta -adrenoreceptor/camp/protein kinase a pathway involving src but independently of erk1/2. J Biol Chem 2000;275:13802-13811.

31 Gomes-Giacoia E, Miyake M, Goodison S, Rosser CJ: Targeting plasminogen activator inhibitor-1 inhibits angiogenesis and tumor growth in a human cancer xenograft model. Mol Cancer Ther 2013;12:2697-2708.

-32 Matsui T, Nishino Y, Maeda S, Yamagishi S: Pedf-derived peptide inhibits corneal angiogenesis by suppressing vegf expression. Microvasc Res 2012;84:105-108.

-33 Lakeland TV, Borg ML, Matzaris M, Abdelkader A, Evans RG, Watt MJ: Augmented expression and secretion of adipose-derived pigment epithelium-derived factor does not alter local angiogenesis or contribute to the development of systemic metabolic derangements. Am J Physiol Endocrinol Metab 2014;306:E1367-1377. 


\section{Cellular Physiology Cell Physiol Biochem 2017;42:974-986 \begin{tabular}{l|l|l} 
DOI: 10.1159/000478680 & O 2017 The Author(s). Published by S. Karger AG, Basel \\
www.karger.com/cpb
\end{tabular}

34 Devy L, Blacher S, Grignet-Debrus C, Bajou K, Masson V, Gerard RD, Gils A, Carmeliet G, Carmeliet P, Declerck PJ, Noel A, Foidart JM: The pro- or antiangiogenic effect of plasminogen activator inhibitor 1 is dose dependent. FASEB J 2002;16:147-154.

-35 Wang QA, Tao C, Gupta RK, Scherer PE: Tracking adipogenesis during white adipose tissue development, expansion and regeneration. Nat Med 2013;19:1338-1344.

-36 Zhang QX, Magovern CJ, Mack CA, Budenbender KT, Ko W, Rosengart TK: Vascular endothelial growth factor is the major angiogenic factor in omentum: Mechanism of the omentum-mediated angiogenesis. J Surg Res 1997;67:147-154.

37 He G, Pedersen SB, Bruun JM, Lihn AS, Jensen PF, Richelsen B: Differences in plasminogen activator inhibitor 1 in subcutaneous versus omental adipose tissue in non-obese and obese subjects. Horm Metab Res 2003;35:178-182.

38 Falcao-Pires I, Castro-Chaves P, Miranda-Silva D, Lourenco AP, Leite-Moreira AF: Physiological, pathological and potential therapeutic roles of adipokines. Drug Discov Today 2012;17:880-889.

39 Nicosia RF, Lin YJ, Hazelton D, Qian X: Endogenous regulation of angiogenesis in the rat aorta model. Role of vascular endothelial growth factor. Am J Pathol 1997;151:1379-1386.

40 Song SY, Chung HM, Sung JH: The pivotal role of vegf in adipose-derived-stem-cell-mediated regeneration. Expert Opin Biol Ther 2010;10:1529-1537.

41 Dohgu S, Takata F, Matsumoto J, Oda M, Harada E, Watanabe T, Nishioku T, Shuto H, Yamauchi A, Kataoka Y: Autocrine and paracrine up-regulation of blood-brain barrier function by plasminogen activator inhibitor-1. Microvasc Res 2011;81:103-107. 